\title{
A general non-adiabatic quantum instanton approximation
}

Cite as: J. Chem. Phys. 152, 204117 (2020); https://doi.org/10.1063/5.0009109

Submitted: 27 March 2020 . Accepted: 11 May 2020 . Published Online: 27 May 2020

(iD) Joseph E. Lawrence, and (iD) David E. Manolopoulos

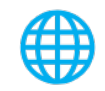

\section{ARTICLES YOU MAY BE INTERESTED IN}

Instanton formulation of Fermi's golden rule in the Marcus inverted regime

The Journal of Chemical Physics 152, 034106 (2020); https://doi.org/10.1063/1.5137823

On the calculation of quantum mechanical electron transfer rates

The Journal of Chemical Physics 151, 114119 (2019); https://doi.org/10.1063/1.5116800

An analysis of isomorphic RPMD in the golden rule limit

The Journal of Chemical Physics 151, 244109 (2019); https://doi.org/10.1063/1.5138913

\section{Challenge us.}

What are your needs for periodic signal detection?

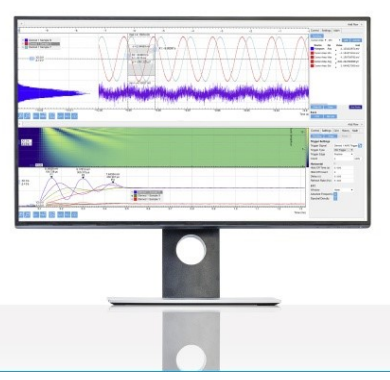

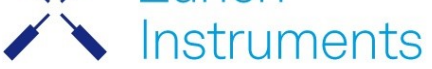




\title{
A general non-adiabatic quantum instanton approximation
}

\author{
Cite as: J. Chem. Phys. 152, 204117 (2020); doi: 10.1063/5.0009109 \\ Submitted: 27 March 2020 - Accepted: 11 May 2020 • \\ Published Online: 27 May 2020
}

Joseph E. Lawrence ${ }^{\text {a) }}$ (D) and David E. Manolopoulos

\begin{abstract}
AFFILIATIONS
Department of Chemistry, University of Oxford, Physical and Theoretical Chemistry Laboratory, South Parks Road, Oxford OX1 3QZ, United Kingdom
\end{abstract}

a) Author to whom correspondence should be addressed: joseph.lawrence@chem.ox.ac.uk

\begin{abstract}
We present a general quantum instanton approach to calculating reaction rates for systems with two electronic states and arbitrary values of the electronic coupling. This new approach, which we call the non-adiabatic quantum instanton (NAQI) approximation, reduces to Wolynes theory in the golden rule limit and to a recently proposed projected quantum instanton method in the adiabatic limit. As in both of these earlier theories, the NAQI approach is based on making a saddle point approximation to the time integral of a reactive flux autocorrelation function, although with a generalized definition of the projection operator onto the product states. We illustrate the accuracy of the approach by comparison with exact rates for one dimensional scattering problems and discuss its applicability to more complex reactions.
\end{abstract}

Published under license by AIP Publishing. https://doi.org/10.1063/5.0009109

\section{INTRODUCTION}

Rate processes that involve both nuclear quantum effects and the breakdown of the Born-Oppenheimer approximation are important in a wide variety of contexts, ranging from device physics to biology. ${ }^{1-4}$ For systems with more than a few degrees of freedom, exact wavefunction based approaches are impractical due to the exponential scaling of quantum mechanics with dimensionality. Imaginary time path integral techniques, ${ }^{5}$ which can accurately capture zero point energy and tunneling effects and yet scale only linearly with system size, have therefore become popular for studying more complex reactions. While there now exist several such methods that are routinely used to study electronically adiabatic reactions, ${ }^{6-12}$ as well as theories that can be applied to electronically non-adiabatic reactions in the golden rule limit, ${ }^{13-20}$ the development of accurate path integral techniques for more general electronically non-adiabatic reactions with intermediate electronic coupling strengths is still a very active area of research. ${ }^{21-33}$

When developing new path integral methods for studying nonadiabatic systems, a common approach has been to generalize a pre-existing adiabatic method. One of the most successful path integral techniques for electronically adiabatic reactions is ring polymer molecular dynamics ${ }^{34,35}$ (RPMD) reaction rate theory, ${ }^{9,10}$ and because of this, much of the work in this area has been focused on trying to extend it to treat non-adiabatic systems. ${ }^{21-31}$ RPMD is particularly effective in the deep tunneling regime because of its connection with the semiclassical instanton approximation, ${ }^{36}$ which uses a periodic imaginary time trajectory through the reaction barrier to describe the tunneling process. ${ }^{37}$ While it is not as generally applicable as RPMD, the semiclassical instanton formula is known to provide a highly accurate description of tunneling in situations where there is a single dominant tunneling path. Early work extending the semiclassical instanton approach to treat non-adiabatic reactions was based on assuming that the "Im-F" premise ${ }^{38,39}$ could be applied to non-adiabatic systems, and succeeded in providing a theory that bridged between the golden rule and Born-Oppenheimer limits. ${ }^{41-44}$ More recently, Richardson et al. ${ }^{45-47}$ have provided a rigorous derivation of the semiclassical instanton rate in the golden rule limit and found some important differences between the resulting expression and that given by the Im-F formulation. However, their derivation has yet to be extended beyond the golden rule limit so that it can be applied to reactions with arbitrary electronic coupling strengths.

In this paper, we shall focus on another well-known method, the quantum instanton approximation. Unlike the other methods discussed above, this approximation was, in fact, first suggested in 
the golden rule context by Wolynes ${ }^{13}$ in 1987 (leading to what is now typically referred to as Wolynes theory), before the adiabatic counterpart was suggested by Miller et al. ${ }^{7}$ in 2003 (who gave it the name quantum instanton). Wolynes theory and the quantum instanton are both closely related to the semiclassical instanton, and all three can be interpreted as steepest descent approximations to the flux-flux correlation function expression for the reaction rate. However, whereas the semiclassical instanton simultaneously approximates integrals over both position and time, Wolynes theory and the quantum instanton involve just a single steepest descent approximation to the time integral. The resulting expressions only involve time-independent quantities, which can be evaluated by sampling imaginary time paths.

Recently, Vaillant et al. ${ }^{48}$ have suggested a slight modification of the original adiabatic quantum instanton, which they have called the projected quantum instanton (PQI). This enforces sampling of paths close to the semiclassical instanton and results in an expression that is even more closely related to Wolynes theory. In the following, we shall present a generalized approach to electronically nonadiabatic reactions that is applicable to arbitrary electronic coupling strengths between the golden rule and adiabatic limits and which reduces to Wolynes theory and the PQI approximation in these two limits, respectively.

We begin in Sec. II by discussing how the choice of the projection operators that are used to define the reactants and products affects the functional form of the reactive flux-flux correlation function of an electronically non-adiabatic reaction. Motivated by this discussion, we introduce a simple projection operator onto the product states, which can be tuned so as to minimize the recrossing of the transition state dividing surface for any given non-adiabatic reaction. In Sec. III, we summarize the Wolynes theory and quantum instanton approaches to the golden rule and Born-Oppenheimer limits before introducing a more general approach for the calculation of reaction rates for arbitrary electronic coupling strengths, which we shall call the non-adiabatic quantum instanton (NAQI) approximation. In Sec. IV, we investigate the accuracy of the NAQI formula for a series of simple one-dimensional scattering problems for which the exact quantum mechanical reaction rates can be computed for comparison. Section $V$ concludes the paper, discussing several possible applications of the NAQI approach and the scope for further theoretical developments.

\section{EXACT REACTION RATE THEORY}

The Hamiltonian for a general two level system can be written in the diabatic representation as

$$
\hat{H}=\hat{H}_{0}|0\rangle\left\langle 0\left|+\hat{H}_{1}\right| 1\right\rangle\langle 1|+\Delta(|0\rangle\langle 1|+| 1\rangle\langle 0|),
$$

where $|0\rangle$ and $|1\rangle$ are the two diabatic electronic states. Since it suffices for our present purposes, we shall restrict our attention to simple one-dimensional scattering problems of the form

$$
\hat{H}_{i}=\frac{\hat{p}^{2}}{2 m}+V_{i}(\hat{q})
$$

in which $V_{i}(q)$ is the diabatic potential on electronic state $|i\rangle$. We shall also assume that the electronic coupling $\Delta$ is a constant, independent of the nuclear configuration $q$ (the Condon approximation). Within this simple framework, the adiabatic potentials, $U_{ \pm}(q)$, are

$$
U_{ \pm}(q)=\frac{V_{0}(q)+V_{1}(q)}{2} \pm \frac{1}{2} \sqrt{\left(V_{0}(q)-V_{1}(q)\right)^{2}+4 \Delta^{2}} .
$$

Everything we shall have to say can readily be generalized to treat more complex multi-dimensional reactions and to include nonCondon effects. However, Eqs. (1)-(3) are all we shall need to make the points we would like to make here.

The exact quantum mechanical thermal rate constant for the transition from reactants to products can be written in the form ${ }^{49,50}$

$$
k Q_{r}=\frac{1}{2} \int_{-\infty}^{\infty} c_{\mathrm{ff}}(t) \mathrm{d} t
$$

where $Q_{r}=\sqrt{m / 2 \pi \beta \hbar^{2}}$ is the reactant partition function per unit length and

$$
c_{\mathrm{ff}}(t)=\operatorname{tr}\left[e^{-\beta \hat{H} / 2} \hat{F} e^{-\beta \hat{H} / 2} e^{+i \hat{H} t / \hbar} \hat{F} e^{-i \hat{H} t / \hbar}\right]
$$

is a reactive flux autocorrelation function (with $\beta=1 / k_{\mathrm{B}} T$ ). The flux operator is the Heisenberg time derivative of the projection onto the products,

$$
\hat{F}=\frac{i}{\hbar}\left[\hat{H}, \hat{P}_{p}\right] .
$$

This formulation applies equally well in both the adiabatic and non-adiabatic limits with appropriate definitions of the projection operator $\hat{P}_{p}$.

In the adiabatic limit where the Born-Oppenheimer approximation is valid, it is usual to define $\hat{P}_{p}$ as

$$
\hat{P}_{p}=\theta(s(\hat{q})),
$$

where $\theta(x)$ is a Heaviside step function and $s(q)=0$ is a position space dividing surface between the reactants $[s(q)<0]$ and products $[s(q)>0]$. The flux operator then becomes

$$
\hat{F}=\frac{\hat{p}}{2 m} \frac{\partial s}{\partial q} \delta(s(\hat{q}))+\frac{\partial s}{\partial q} \delta(s(\hat{q})) \frac{\hat{p}}{2 m} .
$$

In the golden rule limit where $\Delta \rightarrow 0$, it is more usual to define the rate in terms of a transition between the diabatic states $|0\rangle$ and $|1\rangle$, which gives

$$
\hat{P}_{p}=|1\rangle\langle 1|
$$

and

$$
\hat{F}=\frac{i}{\hbar} \Delta(|0\rangle\langle 1|-| 1\rangle\langle 0|) .
$$

In situations where the reactants and products can be equally well distinguished using either definition of $\hat{P}_{p}$, the rate constant is independent of the definition used. However, while the choice of projection operator does not change the rate, it does change the functional form of the flux-flux correlation function. To illustrate this, we shall consider a simple model curve crossing problem, 


$$
\begin{aligned}
& V_{0}(q)=A e^{+q / L}, \\
& V_{1}(q)=A e^{-q / L},
\end{aligned}
$$

with $\beta A=48, m L^{2} / \beta \hbar^{2}=1 / 4$, and three different values of the electronic coupling strength $(\beta \Delta)$.

Figure 1 shows the flux-flux correlation functions for this model problem with $\log _{10}(\beta \Delta)=1.5$ (approximately in the adiabatic limit), as calculated using both the adiabatic and non-adiabatic projection operators. The position space projection operator leads to a correlation function which decays quickly with almost no negative correlation, whereas the diabatic projection operator gives a correlation function that is much larger at $t=0$ and has a slowly decaying negative tail. The position space projection operator is thus seen to lead to far less recrossing than the diabatic projection operator in this regime.

Figure 2 shows the flux-flux correlation function for $\log _{10}(\beta \Delta)$ $=-2$, which is in the opposite (non-adiabatic) limit. In this case, the amount of recrossing is reversed, with the diabatic projection operator leading to an approximately Gaussian flux-flux correlation function with minimal recrossing, whereas the position space projection operator gives rise to significant recrossing. Here, the recrossing arises from the high probability that when the system passes through the dividing surface, it will remain on the same diabatic surface and hence will, a short time later, be reflected by the potential wall and return through the dividing surface.

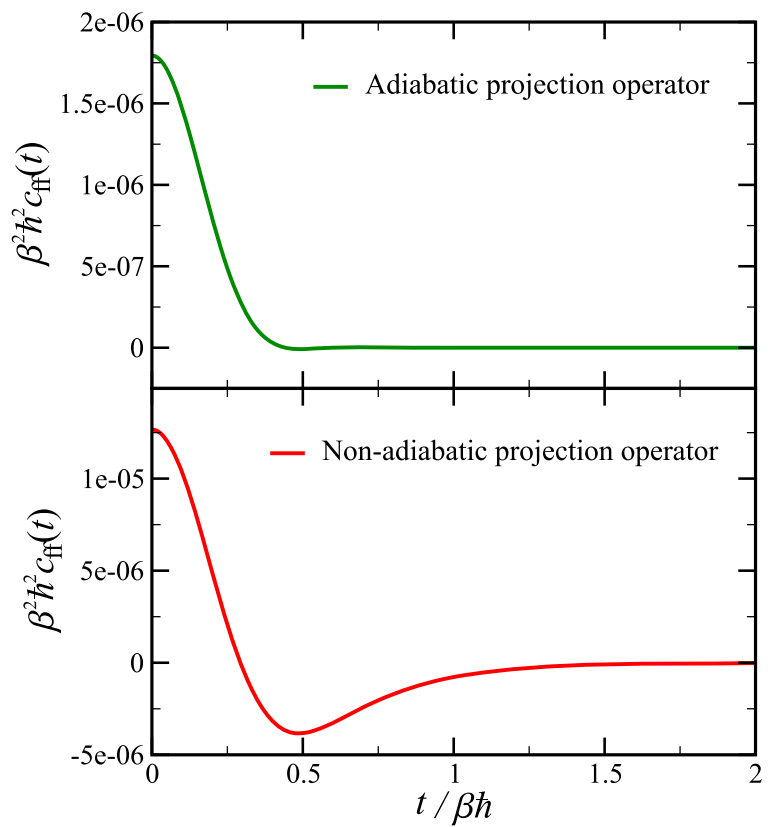

FIG. 1. Flux-flux correlation functions for an exponential crossing model in the adiabatic regime [with $\beta A=48, m L^{2} / \beta \hbar^{2}=1 / 4$, and $\log _{10}(\beta \Delta)=1.5$ ]. Note the different scales on the $y$-axes in the two panels. The areas under both curves are the same. However, the non-adiabatic projection operator clearly leads to a much longer-lived correlation function with a negative tail, which indicates recrossing of the dividing surface between reactants and products.

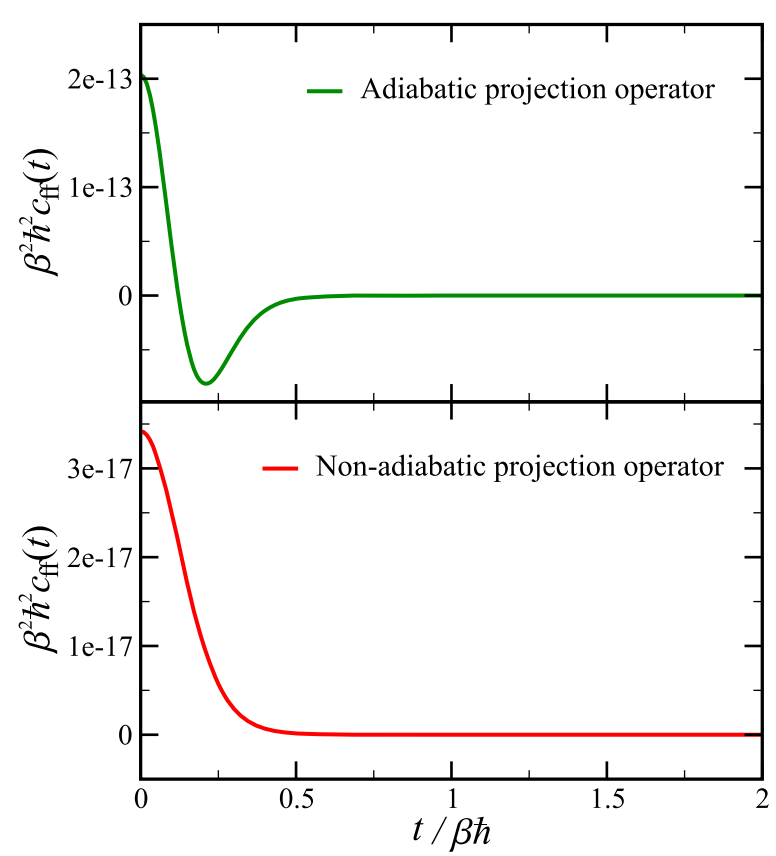

FIG. 2. Flux-flux correlation functions for an exponential crossing model in the golden rule regime [with $\beta A=48, m L^{2} / \beta \hbar^{2}=1 / 4$, and $\log _{10}(\beta \Delta)=-2$ ]. Note the different scales on the $y$-axes in the two panels. The areas under both curves are the same. However, the adiabatic projection operator clearly leads to a correlation function with a negative tail, which indicates recrossing of the dividing surface between reactants and products.

The projection operators in Eqs. (7) and (9) are clearly quite different. However, we note that it is possible to recast the projection operator on to the products in the golden rule limit in a form that more closely resembles Eq. (7),

$$
\hat{P}_{p}=\theta\left(-\hat{\sigma}_{z}\right),
$$

where $\hat{\sigma}_{z}$ is the Pauli spin operator, $\hat{\sigma}_{z}=|0\rangle\langle 0|-| 1\rangle\langle 1|$. This suggests an obvious generalization in which the argument of the Heaviside step function is taken to be a linear combination of $s(\hat{q})$ and $\hat{\sigma}_{z}$,

$$
\hat{P}_{p}(\alpha)=\theta\left(\cos (\alpha) s(\hat{q})-\sin (\alpha) \hat{\sigma}_{z}\right),
$$

or equivalently, provided $0 \leq \alpha<\pi / 2$, using the properties of the step function,

$$
\hat{P}_{p}(\alpha)=\theta\left(s(\hat{q})-\tan (\alpha) \hat{\sigma}_{z}\right) .
$$

This $\hat{P}_{p}(\alpha)$ is diagonal in the electronic basis with diagonal matrix elements,

$$
\begin{aligned}
& \left\langle 0\left|\hat{P}_{p}(\alpha)\right| 0\right\rangle=\theta(s(\hat{q})-\tan (\alpha)), \\
& \left\langle 1\left|\hat{P}_{p}(\alpha)\right| 1\right\rangle=\theta(s(\hat{q})+\tan (\alpha)),
\end{aligned}
$$

and so, we see that we can also think of the generalized projection operator as effectively giving rise to two separate position space dividing surfaces, one for each diabatic state.

In the Born-Oppenheimer limit, it is clear that $\alpha \rightarrow 0$ and in the non-adiabatic limit that $\alpha \rightarrow \pi / 2$. In Fig. 3, we show the flux-flux correlation functions for the system with $\log _{10}(\beta \Delta)=-0.25$, which is intermediate between the adiabatic and non-adiabatic limits. The 


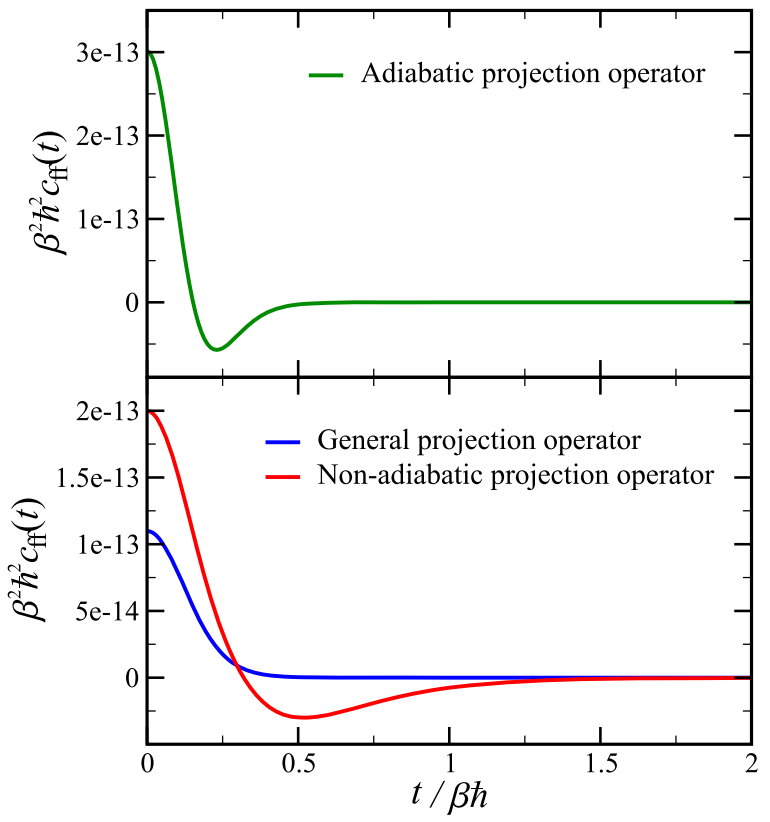

FIG. 3. Flux-flux correlation functions for an exponential crossing model with parameters intermediate between the golden rule and the adiabatic limits $\left[\beta A=48, m L^{2} / \beta \hbar^{2}=1 / 4\right.$, and $\left.\log _{10}(\beta \Delta)=-0.25\right]$. Note the different scales on the $y$-axes in the two panels. The plot clearly illustrates that in this regime, the adiabatic projection operator leads to a correlation function with significant recrossing of the dividing surface and the non-adiabatic projection operator leads to a much longer lived correlation function with a negative tail. The generalized projection operator is seen to give a correlation function with almost no recrossing.

results for all three projection operators are shown, with $s(q)=q / L$ and $\tan (\alpha)=12 / 5$ in the case of the generalized projection operator in Eq. (14). We see that with this choice of $\alpha$, the correlation function is approximately Gaussian with minimal recrossing. In contrast, both the purely position space and purely diabatic state projection operators have significantly higher initial values along with regions of negative correlation corresponding to recrossing.

\section{QUANTUM TRANSITION STATE THEORIES}

The advantage of writing the rate in terms of the integral of a flux-flux correlation function with minimal recrossing is that one can then use this to develop a "quantum-transition state theory." By this, we mean an approximate expression for the rate that only depends on time-independent quantities, such as Wolynes theory in the non-adiabatic limit ${ }^{13}$ and the quantum instanton approximation in the adiabatic limit. ' Here, we first give a brief summary of these two existing quantum transition state theories before showing how they can be generalized to give a method that is applicable to electronically non-adiabatic reactions with arbitrary electronic coupling strengths.

\section{A. Wolynes theory}

In order to derive the Wolynes theory expression for the rate, one begins by taking the golden rule limit of the flux-flux correlation function in Eq. (5), with the flux operator defined as in Eq. (10). Since $\left\langle i\left|e^{-i \hat{H} t / \hbar}\right| i\right\rangle=e^{-i \hat{H}_{i} t / \hbar}+\mathcal{O}\left(\Delta^{2}\right)$ and $\left\langle i\left|e^{-i \hat{H} t / \hbar}\right| j\right\rangle=\mathcal{O}(\Delta)$ for $i \neq j$, in the golden rule $(\Delta \rightarrow 0)$ limit,

$$
c_{\mathrm{ff}}(t)=\frac{2 \Delta^{2}}{\hbar^{2}} \operatorname{Re}\left(c_{\mathrm{GR}}(t)\right),
$$

where

$$
c_{\mathrm{GR}}(t)=\operatorname{tr}_{\mathrm{n}}\left[e^{-\beta \hat{H}_{0} / 2-i \hat{H}_{0} t / \hbar} e^{-\beta \hat{H}_{1} / 2+i \hat{H}_{1} t / \hbar}\right]
$$

and $\operatorname{tr}_{\mathrm{n}}[\cdots]$ denotes a trace over nuclear coordinates. Noting that introducing an arbitrary bias to products, $V_{1}(q) \rightarrow V_{1}(q)-\epsilon$, leads to

$$
c_{\mathrm{GR}}(t) \rightarrow c_{\mathrm{GR}}(t) e^{+\beta \epsilon / 2-i \epsilon t / \hbar},
$$

it is clear that changing the bias introduces an oscillatory component into the correlation function. In order to remove this oscillation and restore the approximately Gaussian behavior seen for the symmetric problem in the lower panel of Fig. 2, one makes use of the relation $c_{\mathrm{GR}}(-t)=c_{\mathrm{GR}}^{*}(t)$ to rewrite the rate in Eq. (4) as

$$
k Q_{r}=\frac{\Delta^{2}}{\hbar^{2}} \int_{-\infty}^{\infty} c_{\mathrm{GR}}(t) \mathrm{d} t
$$

and then performs the integration over time by shifting the contour of integration to pass through a saddle point of $c_{\mathrm{GR}}(t)$ on the imaginary time axis. This leads to the Wolynes theory approximation to the rate ${ }^{13}$

$$
k_{\mathrm{WT}} Q_{r}=\frac{\Delta^{2}}{\hbar} \sqrt{\frac{2 \pi}{-\beta F_{\mathrm{WT}}^{\prime \prime}\left(\lambda_{\mathrm{sp}}\right)}} e^{-\beta F_{\mathrm{WT}}\left(\lambda_{\mathrm{sp}}\right)},
$$

where we have defined

$$
\begin{aligned}
e^{-\beta F_{\mathrm{WT}}(\lambda)} & =\operatorname{tr}_{\mathrm{n}}\left[e^{-(\beta-\lambda) \hat{H}_{0}} e^{-\lambda \hat{H}_{1}}\right] \\
& =c_{\mathrm{GR}}(i(\lambda-\beta / 2) \hbar)
\end{aligned}
$$

and the saddle point condition is $F_{\mathrm{WT}}^{\prime}\left(\lambda_{\mathrm{sp}}\right)=0$.

\section{B. Adiabatic quantum instanton}

In the adiabatic limit, for reactions which can be considered to proceed on the lower adiabatic surface, the flux-flux correlation function becomes

$$
c_{\mathrm{ff}}(t)=\operatorname{tr}_{\mathrm{n}}\left[e^{-\beta \hat{H}_{\mathrm{BO}} / 2} \hat{F} e^{-\beta \hat{H}_{\mathrm{BO}} / 2} e^{+i \hat{H}_{\mathrm{BO}} t / \hbar} \hat{F} e^{-i \hat{H}_{\mathrm{BO}} t / \hbar}\right],
$$

where

$$
\hat{H}_{\mathrm{BO}}=\frac{\hat{p}^{2}}{2 m}+\hat{U}_{-}(q)
$$

and the flux operator is

$$
\hat{F}=\frac{i}{\hbar}\left[\hat{H}_{\mathrm{BO}}, \hat{P}_{p}\right]
$$

The original paper by Miller et al. ${ }^{7}$ proposed two closely related "quantum instanton" methods. The conceptually simpler of the two makes a second order cumulant approximation to the flux-flux 
correlation function,

$$
c_{\mathrm{fff}}(t) \simeq c_{\mathrm{fff}}(0) \exp \left(\frac{\ddot{c}_{\mathrm{ff}}(0)}{2 c_{\mathrm{ff}}(0)} t^{2}\right)
$$

and then integrates over time to give

$$
k_{\mathrm{QI}} Q_{r}=\sqrt{\frac{\pi c_{\mathrm{ff}}(0)}{-2 \ddot{c}_{\mathrm{ff}}(0)}} c_{\mathrm{cff}}(0) .
$$

The problem with this approach is that the second order cumulant expansion of the flux-flux correlation function can be a poor approximation for asymmetric reactions. This can be understood as arising for essentially the same reason as we have discussed above for Wolynes theory. Vaillant et al. ${ }^{48}$ have recently examined the problem in detail with a semiclassical analysis in which they showed that neither Eq. (26) nor the alternative formulation of the quantum instanton method in the original paper by Miller et al. reduces to the semiclassical instanton approximation in the limit as $\hbar \rightarrow 0$.

In order to fix this problem, Vaillant et al. have suggested a modified method, the projected quantum instanton (PQI), in which the flux-flux correlation function is approximated as ${ }^{48}$

$$
c_{\mathrm{ff}}(t) \simeq 2 \operatorname{Re}\left[c_{\mathrm{PQI}}(t)\right],
$$

where

$$
c_{\mathrm{PQI}}(t)=\operatorname{tr}_{n}\left[U_{r}(i \beta \hbar / 2-t) \hat{F} U_{p}(i \beta \hbar / 2+t) \hat{F}\right],
$$

with

$$
U_{s}(t)=e^{+i \hat{H}_{\mathrm{BO}} t /(2 \hbar)} \hat{P}_{s} e^{+i \hat{H}_{\mathrm{BO}} t /(2 \hbar)}
$$

for $s=r$ and $p$, with $\hat{P}_{r}=\hat{1}-\hat{P}_{p}$. The difference between Eqs. (22) and (27) is that the exact $c_{\mathrm{ff}}(t)$ contains two additional terms of the form

$$
\operatorname{tr}_{n}\left[U_{s}(i \beta \hbar / 2-t) \hat{F} U_{s}(i \beta \hbar / 2+t) \hat{F}\right],
$$

with $s=r$ and $p$. However, Vaillant et al. argued that since the time integrals of these terms vanish in the semiclassical $(\hbar \rightarrow 0)$ limit, they can be safely neglected when calculating the reaction rate. ${ }^{48}$

Substituting Eq. (27) into Eq. (4) and noting that $c_{\mathrm{PQI}}(-t)$ $=c_{\mathrm{PQI}}^{*}(t)$ gives

$$
k Q_{r} \simeq \int_{-\infty}^{\infty} c_{\mathrm{PQI}}(t) \mathrm{d} t .
$$

This time integral can be evaluated by steepest descent as in Wolynes theory to give

$$
k_{\mathrm{PQI}} Q_{r}=\frac{1}{\beta^{2} \hbar} \sqrt{\frac{2 \pi}{-\beta F_{\mathrm{PQI}}^{\prime \prime}\left(\lambda_{\mathrm{sp}}\right)}} e^{-\beta F_{\mathrm{PQI}}\left(\lambda_{\mathrm{sp}}\right)},
$$

where

$$
\begin{aligned}
e^{-\beta F_{\mathrm{PQI}}(\lambda)} & =(\beta \hbar)^{2} \operatorname{tr}_{n}\left[U_{r}(i(\beta-\lambda) \hbar) \hat{F} U_{p}(i \lambda \hbar) \hat{F}\right] \\
& =(\beta \hbar)^{2} c_{\mathrm{PQI}}(i(\lambda-\beta / 2) \hbar),
\end{aligned}
$$

in which the factor of $(\beta \hbar)^{2}$ has been introduced to ensure dimensional consistency in Eq. (32) and then compensated for in Eq. (31). The saddle point condition is now $F_{\mathrm{PQI}}^{\prime}\left(\lambda_{\mathrm{sp}}\right)=0$, which is satisfied by the value of $\lambda$ that maximizes $F_{\mathrm{PQI}}(\lambda)$ and minimizes $c_{\mathrm{PQI}}(i(\lambda-\beta / 2) \hbar)$.

\section{Non-adiabatic quantum instanton}

It is clear from the above discussion that the PQI method is very closely related to Wolynes theory. ${ }^{13}$ This connection can be made more explicit by noting that Wolynes theory and the PQI method can be regarded as the golden rule limit and adiabatic limit, respectively, of a more general non-adiabatic QI method.

In order to derive this NAQI method, one simply proceeds as in Eqs. (27)-(32), but with the flux operator defined as in Eq. (6) with the generalized projection operator $\hat{P}_{p}(\alpha)$ in Eq. (14). The final result has the same form as Eq. (31),

$$
k_{\mathrm{NAQI}} Q_{r}=\frac{1}{\beta^{2} \hbar} \sqrt{\frac{2 \pi}{-\beta F_{\alpha^{*}}^{\prime \prime}\left(\lambda_{\mathrm{sp}}\right)}} e^{-\beta F_{\alpha^{\star}}\left(\lambda_{\mathrm{sp}}\right)} \text {, }
$$

where

$$
\begin{aligned}
e^{-\beta F_{\alpha}(\lambda)} & =(\beta \hbar)^{2} \operatorname{tr}_{n}\left[U_{r}(i(\beta-\lambda) \hbar) \hat{F} U_{p}(i \lambda \hbar) \hat{F}\right] \\
& =(\beta \hbar)^{2} c_{\alpha}(i(\lambda-\beta / 2) \hbar),
\end{aligned}
$$

and we have defined $\alpha^{*}$ and $\lambda_{\mathrm{sp}}$ as the values of $\alpha$ and $\lambda$ that maximize $F_{\alpha}(\lambda)$ and minimize $c_{\alpha}(i(\lambda-\beta / 2) \hbar)$.

When $\Delta \rightarrow 0$ and $\alpha^{*} \rightarrow \pi / 2$, as is the case in the golden rule limit, Eq. (33) reduces to Wolynes theory, ${ }^{13}$ and when the upper adiabatic electronic state becomes thermally inaccessible and $\alpha^{*} \rightarrow 0$, it reduces to the adiabatic PQI of the study of Vaillant et al. ${ }^{48}$ The NAQI method is thus a generalization of these pre-existing methods, which can be applied to reactions that are intermediate between the two limiting regimes.

\section{RESULTS AND DISCUSSION}

To illustrate the accuracy of the NAQI approach, we shall consider a simple generalization of the exponential curve crossing model from Sec. II,

$$
\begin{gathered}
V_{0}(q)=A e^{+q / L}, \\
V_{1}(q)=A e^{-q / L}-\epsilon,
\end{gathered}
$$

which allows for a bias of $\epsilon$ toward the products. Defining the crossing point of the two diabats as the solution to the equation $V_{0}\left(q^{\ddagger}\right)$ $=V_{1}\left(q^{\ddagger}\right)$ and the value of the potential at the crossing point as $V^{\ddagger}=V_{0}\left(q^{\ddagger}\right)$, the behavior of this model can be fully characterized by the four dimensionless parameters, $\beta \Delta, \beta V^{\ddagger}, \beta \epsilon$, and $m L^{2} / \beta \hbar^{2}$. Note that $A=\sqrt{\left(V^{\ddagger}+\epsilon / 2\right)^{2}-\epsilon^{2} / 4}$.

To demonstrate the behavior of the NAQI method in different regimes, we shall consider three systems in which the values of $\beta V^{\ddagger}, \beta \epsilon$, and $m L^{2} / \beta \hbar^{2}$ are fixed, while $\beta \Delta$ is varied so as to span the range from golden rule to Born-Oppenheimer-like behavior. For each value of $\beta \Delta$, the NAQI rate was evaluated using a sine finite basis representation (FBR) in the barrier region, with $\alpha$ and $\lambda$ 
optimized along with the location of the position space dividing surface, $s(q)=\left(q-q_{0}\right) / L$, so as to minimize $c_{\alpha}(i(\lambda-\beta / 2) \hbar)$. The exact rate was computed for comparison by integrating the cumulative reaction probability

$$
k Q_{r} \beta \hbar=\frac{1}{2 \pi} \int_{0}^{\infty} e^{-\beta E} N(E) \beta \mathrm{d} E,
$$

with $N(E)$ calculated using the coupled channel log derivative method.

In order to illustrate the importance of nuclear quantum effects, we shall compare the exact and NAQI results with the classical Born-Oppenheimer rate,

$$
k_{\mathrm{cl}-\mathrm{BO}} Q_{r} \beta \hbar=\frac{1}{2 \pi} e^{-\beta U_{-}^{\ddagger}},
$$

where $U_{-}^{\ddagger}=\frac{1}{2}\left(\sqrt{4(A-\Delta)^{2}+\epsilon^{2}}-\epsilon\right)$ is the maximum on the lower adiabatic potential, and with the classical golden rule rate,

$$
k_{\mathrm{cl}-\mathrm{GR}} Q_{r} \beta \hbar=\frac{\Delta^{2} \sqrt{2 \pi m \beta}}{\hbar\left|V_{0}^{\prime}\left(q^{\ddagger}\right)-V_{1}^{\prime}\left(q^{\ddagger}\right)\right|} e^{-\beta V^{\ddagger}},
$$

where $\left|V_{0}^{\prime}\left(q^{\ddagger}\right)-V_{1}^{\prime}\left(q^{\ddagger}\right)\right|=\left(2 V^{\ddagger}+\epsilon\right) / L$. In terms of the four dimensionless parameters, this is simply

$$
k_{\mathrm{cl}-\mathrm{GR}} Q_{r} \beta \hbar=\frac{\beta^{2} \Delta^{2}}{\left(2 \beta V^{\ddagger}+\beta \epsilon\right)} \sqrt{\frac{2 \pi m L^{2}}{\beta \hbar^{2}}} e^{-\beta V^{\ddagger}} .
$$

The first system we shall consider is the symmetric problem from Sec. II, with $\beta \epsilon=0, \beta V^{\ddagger}=48$, and $m L^{2} / \beta \hbar^{2}=1 / 4$. These parameters were chosen so that when $\log _{10}(\beta \Delta)=1.5$, the BornOppenheimer problem on the lower adiabatic potential is similar to the $300 \mathrm{~K}$ symmetric Eckart barrier problem considered by Vaillant et al. ${ }^{48}$ Figure 4 compares the NAQI rate with the exact rate for this system. Excellent agreement is obtained for the full range of $\beta \Delta$ considered. The error in the NAQI rate is approximately independent of $\beta \Delta$ and is slightly less than $5 \%$. Comparison with the classical golden rule and Born-Oppenheimer rates highlights the importance of nuclear quantum effects, showing, in particular, that the effect of tunneling on the rate is strongly dependent on the electronic coupling strength. The tunneling enhancement of the rate ranges from a factor of 30 at the largest value of electronic coupling, $\log _{10}(\beta \Delta)=1.5$, to 12 orders of magnitude in the golden rule limit. Clearly, there is very efficient tunneling through the narrow, nearly cusped potential energy barrier at small values of the coupling.

It is interesting to note that the rate constant for the intermediate value of coupling considered in Fig. $3, \log _{10}(\beta \Delta)=-0.25$, is actually very well described by the golden rule limit in this system. Hence, we see that the electronic coupling at which the transition from Born-Oppenheimer to golden rule behavior occurs is larger for the rate than the flux-flux correlation function. This results in the optimum value of $\alpha$ remaining close to the adiabatic value, while the rate exhibits an approximately quadratic dependence on the electronic coupling characteristic of the golden rule limit. We do not expect this to be a general feature-it occurs here because this is a symmetric problem in the deep tunneling regime. In fact, we believe that this helps explain the success of "mean field" methods for such problems. ${ }^{24,44}$ These methods exploit the fact that at intermediate

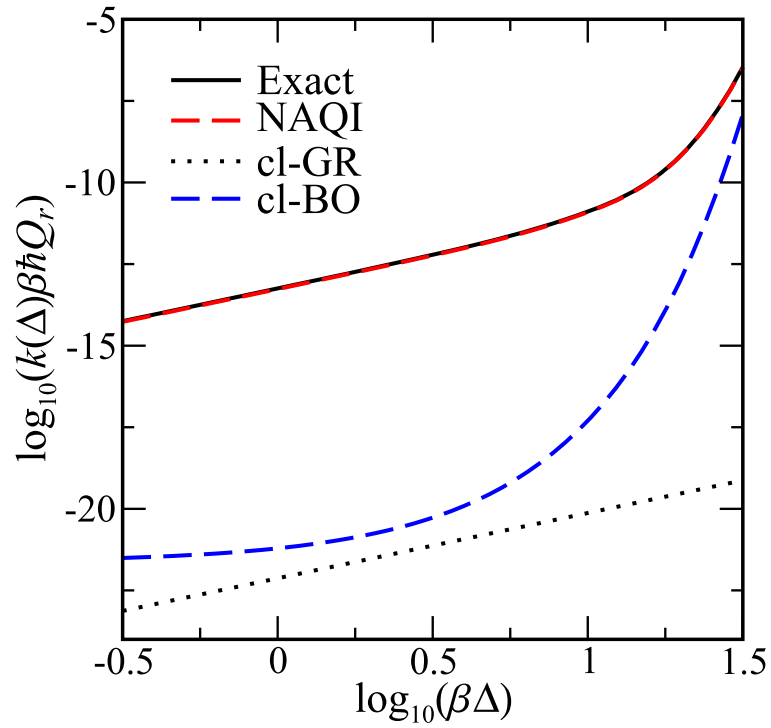

FIG. 4. Comparison of the exact and NAQI rates as a function of the diabatic coupling $\log (\beta \Delta)$ for a symmetric exponential crossing model with $\beta \epsilon=0, \beta V^{\ddagger}$ $=48$, and $m L^{2} / \beta \hbar^{2}=1 / 4$. The classical Born-Oppenheimer and golden rule rates are included to illustrate the importance of nuclear quantum effects.

values of the electronic coupling, the dominant contribution to the partition function in the barrier region is the $\Delta^{2}$ term. The $\Delta^{0}$ terms have a larger action due to lack of tunneling, whereas the $\Delta^{2}$ term is dominated by paths near the golden rule instanton, which only spend a brief amount of time near the crossing point and have a smaller action. Note that the mean field instanton based methods all involve some kind of ad hoc approximation in which the $\Delta^{0}$ term is thrown away, whereas this is unnecessary in the present NAQI approach.

Figure 5 shows the various rates as a function of the electronic coupling for the second system we shall consider, a strongly asymmetric system with $\beta \epsilon=50, \beta V^{\ddagger}=44.5$, and $m L^{2} / \beta \hbar^{2}=16 / 49$. Again the parameters were chosen such that when $\log (\beta \Delta)=1.5$, the lower adiabatic potential is similar to that in one of the systems studied by Vaillant et al., in this case the most asymmetric system with $\alpha=4$ at $300 \mathrm{~K}$ in Fig. 5 of Ref. 48. As with the first system, we see large nuclear quantum effects at all values of electronic coupling, with the largest quantum enhancement in the golden rule limit. We find excellent agreement between the exact rate and the NAQI rate, with the largest error near $\log (\beta \Delta)=-0.5$, where the NAQI rate underestimates the exact rate by around $20 \%$. Interestingly the rate is again well described by Fermi's golden rule at this value of $\beta \Delta$, despite the correlation function and the optimum value of $\alpha$ being intermediate between the Born-Oppenheimer and golden rule regimes. However, in contrast to the symmetric system considered above, the optimum value of $\alpha$ moves significantly away from zero before the rate has begun to exhibit a quadratic dependence on the electronic coupling.

The high accuracy of the method for such an asymmetric system at all values of the electronic coupling is particularly encouraging as it indicates that we are correctly capturing the instanton in this 


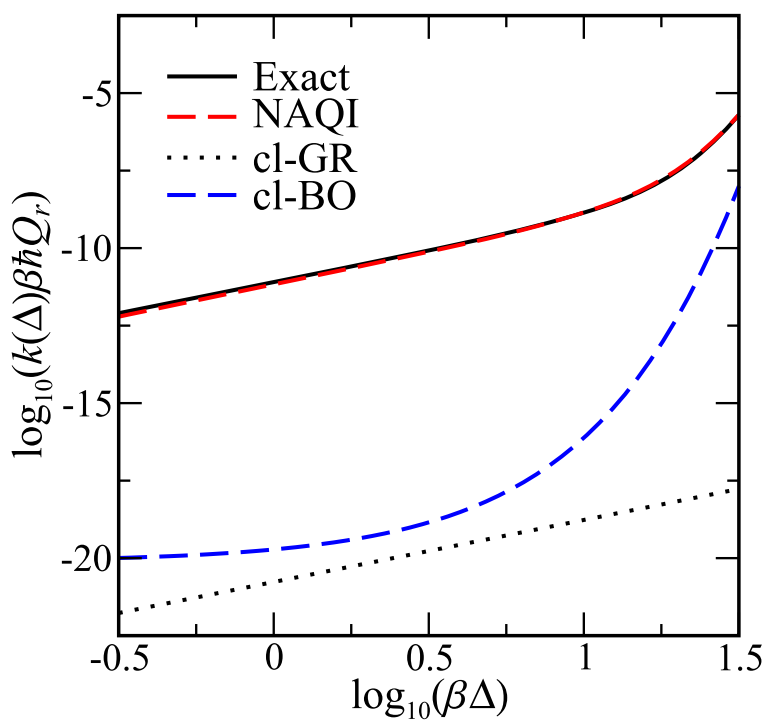

FIG. 5. Comparison of the exact and NAQI rates as a function of the diabatic coupling $\log (\beta \Delta)$ for an asymmetric exponential crossing model with $\beta \epsilon=50$, $\beta V^{\ddagger}=44.5$, and $m L^{2} / \beta \hbar^{2}=16 / 49$. The classical Born-Oppenheimer and golden rule rates are included to illustrate the importance of nuclear quantum effects.

system. In particular, it shows that the introduction of the projection operators into the flux-flux correlation function works not only in the golden rule and adiabatic limits but also at intermediate values of the electronic coupling. In this regard, the present method provides a significant improvement over previous theories, such as the QTST of Schwieters and Voth, ${ }^{43,44}$ which break down for strongly asymmetric systems.

The final system we shall consider is significantly less quantum mechanical while still being strongly asymmetric. This is achieved by increasing the dimensionless mass parameter by a factor of 10 relative to the previous model so that $\beta \epsilon=50, \beta V^{\ddagger}=44.5$, and $m L^{2} / \beta \hbar^{2}$ $=160 / 49$. Figure 6 compares the exact rate with the NAQI rate as well as the classical golden rule and Born-Oppenheimer rates for this system. We see that for the largest values of electronic coupling, the classical adiabatic rate becomes a very good approximation to the exact rate, indicating that nuclear quantum effects are minimal in this regime. The NAQI rate again agrees very closely with the exact rate for all values of the electronic coupling, with errors less than $5 \%$ for $\log _{10}(\beta \Delta)<1$. The largest errors are observed at the largest coupling strengths in the range $1.25 \leq \log _{10}(\beta \Delta) \leq 1.5$. We find that the NAQI approximation underestimates the exact rate by about $40 \%$ at the upper end of this range, where the reaction is approximately classical and adiabatic. This is a well-known deficiency of the adiabatic quantum instanton ${ }^{7,8,48}$ and arises because in the classical limit, the correlation function is not well approximated by a Gaussian due to a long time polynomially decaying tail. Analysis of the free particle correlation function predicts that the adiabatic PQI underestimates the exact rate by $37 \%{ }^{48}$ which is entirely consistent with the error seen here in the NAQI rate. Simple fixes have been suggested in the past to correct the adiabatic quantum instanton for this error, ${ }^{7,8}$ and it may be possible to apply similar fixes to the NAQI.

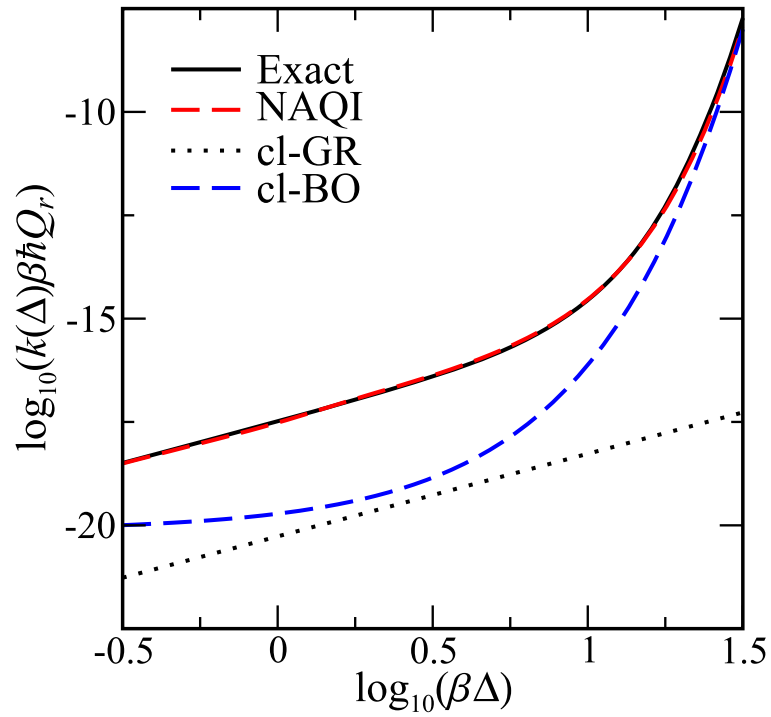

FIG. 6. Comparison of the exact and NAQI rates as a function of the diabatic coupling $\log (\beta \Delta)$ for an asymmetric exponential crossing model with $\beta \epsilon=50$, $\beta V \ddagger=44.5$, and $m L^{2} / \beta \hbar^{2}=160 / 49$. The classical Born-Oppenheimer and golden rule rates are included to illustrate the importance of nuclear quantum effects.

While Wolynes theory and hence the golden rule limit of NAQI reduce to Marcus theory in the high temperature limit for the spinboson model, it is known that they do not give the correct classical result at high temperatures for anharmonic systems. Recently, however, new methods have been suggested which aim to ameliorate this flaw while still accurately describing the low temperature regime, ${ }^{18,19}$ and it may well be possible to generalize these approaches to arbitrary coupling in much the same way we have done here.

\section{CONCLUDING REMARKS}

We have demonstrated that it is straightforward to generalize the PQI approximation and Wolynes theory, which are applicable in the Born-Oppenheimer and golden rule limits, respectively, to treat non-adiabatic reactions with arbitrary electronic coupling strengths. The resulting NAQI approximation has been shown to be highly accurate for both symmetric and strongly asymmetric systems at low temperatures where nuclear quantum effects are important. However, as is expected from its connection with the adiabatic quantum instanton, we find that it underestimates the exact rate by approximately $40 \%$ in the adiabatic limit at high temperature, where the flux-flux correlation function is not well approximated by a Gaussian but instead exhibits a polynomially decaying tail. This problem has previously been overcome by assuming a different functional form for the correlation function or by going to a higher order asymptotic approximation, and these are both interesting avenues to explore to improve the present method. ${ }^{7,53-55}$

Here, we have only applied the NAQI approach to simple onedimensional systems in order to demonstrate the basic features of 
the method. In order to apply the method to multidimensional systems, it will be necessary to develop a path integral implementation, which we expect to be straightforward to do starting from the standard path integral implementation of Wolynes theory. ${ }^{13,17}$ One of the main difficulties associated with the calculation of multidimensional quantum instanton rates in the adiabtatic limit is locating the optimum position space dividing surface, and this difficulty will clearly carry over to the present method. We note, however, that the natural reaction coordinate in a two level system is expected to be the diabatic energy gap and hence for many systems, especially those modeled with empirical valence bond force fields, finding an appropriate dividing surface may not be too much of an issue. (However, the generalization to systems with more than two electronic states will be clearly much more challenging.)

We have recently suggested an alternative approach to calculating non-adiabatic reaction rates, which avoids the need to optimize the dividing surface. ${ }^{32,33}$ The idea is to combine the BornOppenheimer and golden rule rates with an appropriate interpolation formula. When the Born-Oppenheimer rate is calculated using RPMD rate theory ${ }^{9,10}$ and the golden rule rate using Wolynes theory, ${ }^{13}$ the resulting interpolated rate is independent of the choice of position space dividing surface for all values of the electronic coupling strength. ${ }^{32}$ However, the present NAQI approach clearly provides some advantages over the use of an interpolation formula. For example, one could imagine using its path integral implementation to obtain direct information about the imaginary time trajectories that are important in the reaction and how these trajectories change as a function of the electronic coupling.

Finally, we note that by making steepest descent approximations to the integrals over position in PQI and Wolynes theory, one can obtain the semiclassical instanton in the Born-Oppenheimer and golden rule limits, respectively. ${ }^{46,48}$ Hence, we expect that a steepest descent approximation to the NAQI should lead to an accurate semiclassical instanton, which is valid for arbitrary electronic coupling strengths. More speculatively, because the semiclassical instanton in the adiabatic limit has a close connection to (also has been shown to provide an a posteriori justification for) RPMD rate theory, ${ }^{36}$ one might hope that the resulting nonadiabatic semiclassical instanton may help in the development of an accurate generalization of RPMD for calculating non-adiabatic rates.

\section{ACKNOWLEDGMENTS}

The authors thank Jeremy Richardson and Manish Thapa for helpful discussions. J. E. Lawrence is supported by The Queen's College Cyril and Phillis Long Scholarship in conjunction with the Clarendon Fund of the University of Oxford and the EPRSC Centre for Doctoral Training in Theory and Modeling in the Chemical Sciences, EPSRC (Grant No. EP/L015722/1).

\section{APPENDIX: DIABATIC PROJECTION OPERATOR}

The alternative expression for the diabatic projection operator in Eq. (12) can be derived by noting that

$$
\theta\left(-\hat{\sigma}_{z}\right)=\lim _{\epsilon \rightarrow 0_{+}} \frac{1}{2 \pi i} \int_{-\infty}^{\infty} \frac{e^{-i x \hat{\sigma}_{z}}}{x-i \epsilon} \mathrm{d} x
$$

and

$$
e^{-i x \hat{\sigma}_{z}}=e^{-i x}|0\rangle\left\langle 0\left|+e^{+i x}\right| 1\right\rangle\langle 1|
$$

immediately give

$$
\theta\left(-\hat{\sigma}_{z}\right)=|1\rangle\langle 1|
$$

\section{DATA AVAILABILITY}

The data that support the finding of this study are available in the paper itself.

\section{REFERENCES}

${ }^{1}$ S. Hammes-Schiffer and A. A. Stuchebrukhov, Chem. Rev. 110, 6939 (2010)

${ }^{2}$ J. Blumberger, Chem. Rev. 115, 11191 (2015).

${ }^{3}$ H. Oberhofer, K. Reuter, and J. Blumberger, Chem. Rev. 117, 10319 (2017).

${ }^{4}$ W. Dou and J. E. Subotnik, J. Chem. Phys. 148, 230901 (2018).

${ }^{5}$ R. P. Feynman and A. R. Hibbs, Quantum Mechanics and Path Integrals (McGraw-Hill, New York, 1965).

${ }^{6}$ G. A. Voth, D. Chandler, and W. H. Miller, J. Chem. Phys. 91, 7749 (1989).

${ }^{7}$ W. H. Miller, Y. Zhao, M. Ceotto, and S. Yang, J. Chem. Phys. 119, 1329 (2003).

${ }^{8}$ J. Vaníček, W. H. Miller, J. F. Castillo, and F. J. Aoiz, J. Chem. Phys. 123, 054108 (2005).

${ }^{9}$ I. R. Craig and D. E. Manolopoulos, J. Chem. Phys. 122, 084106 (2005).

${ }^{10}$ I. R. Craig and D. E. Manolopoulos, J. Chem. Phys. 123, 034102 (2005).

${ }^{11}$ R. Collepardo-Guevara, I. R. Craig, and D. E. Manolopoulos, J. Chem. Phys. 128, 144502 (2008).

${ }^{12}$ N. Boekelheide, R. Salomon-Ferrer, and T. F. Miller III, Proc. Natl. Acad. Sci. U. S. A. 108, 16159 (2011).

${ }^{13}$ P. G. Wolynes, J. Chem. Phys. 87, 6559 (1987).

${ }^{14}$ C. Zheng, J. A. McCammon, and P. G. Wolynes, Proc. Natl. Acad. Sci. U. S. A. 86, 6441 (1989).

${ }^{15}$ J. S. Bader, R. A. Kuharski, and D. Chandler, J. Chem. Phys. 93, 230 (1990).

${ }^{16}$ C. Zheng, J. A. McCammon, and P. G. Wolynes, Chem. Phys. 158, 261 (1991).

${ }^{17}$ J. E. Lawrence and D. E. Manolopoulos, J. Chem. Phys. 148, 102313 (2018).

${ }^{18}$ M. J. Thapa, W. Fang, and J. O. Richardson, J. Chem. Phys. 150, 104107 (2019).

${ }^{19}$ W. Fang, M. J. Thapa, and J. O. Richardson, J. Chem. Phys. 151, 214101 (2019).

${ }^{20}$ W. Fang, R. A. Zarotiadis and J. O. Richardson, "Revisiting nuclear tunnelling in the aqueous ferrous-ferric electron transfer," Phys. Chem. Chem. Phys. (published online, 2020).

${ }^{21}$ P. Shushkov, R. Li, and J. C. Tully, J. Chem. Phys. 137, 22 A549 (2012).

${ }^{22}$ J. O. Richardson and M. Thoss, J. Chem. Phys. 139, 031102 (2013).

${ }^{23}$ N. Ananth, J. Chem. Phys. 139, 124102 (2013).

${ }^{24}$ J. R. Duke and N. Ananth, Faraday Discuss. 195, 253 (2016).

${ }^{25}$ A. R. Menzeleev, F. Bell, and T. F. Miller III, J. Chem. Phys. 140, 064103 (2014).

${ }^{26}$ J. S. Kretchmer and T. F. Miller III, Faraday Discuss. 195, 191 (2016).

${ }^{27}$ S. N. Chowdhury and P. Huo, J. Chem. Phys. 147, 214109 (2017).

${ }^{28}$ J. S. Kretchmer, N. Boekelheide, J. J. Warren, J. R. Winkler, H. B. Gray, and T. F. Miller III, Proc. Natl. Acad. Sci. U. S. A. 115, 6129 (2018).

${ }^{29}$ X. Tao, P. Shushkov, and T. F. Miller III, J. Chem. Phys. 148, 102327 (2018).

${ }^{30}$ X. Tao, P. Shushkov, and T. F. Miller III, J. Phys. Chem. A 123, 3013 (2019).

${ }^{31}$ J. E. Lawrence and D. E. Manolopoulos, J. Chem. Phys. 151, 244109 (2019).

${ }^{32}$ J. E. Lawrence, T. Fletcher, L. P. Lindoy, and D. E. Manolopoulos, J. Chem. Phys. 151, 114119 (2019).

${ }^{33}$ J. E. Lawrence and D. E. Manolopoulos, Faraday Discuss. 221, 9 (2020).

${ }^{34}$ I. R. Craig and D. E. Manolopoulos, J. Chem. Phys. 121, 3368 (2004).

${ }^{35}$ S. Habershon, D. E. Manolopoulos, T. E. Markland, and T. F. Miller III, Ann. Rev. Phys. Chem. 64, 387 (2013).

${ }^{36}$ J. O. Richardson and S. C. Althorpe, J. Chem. Phys. 131, 214106 (2009)

${ }^{37}$ W. H. Miller, J. Chem. Phys. 62, 1899 (1975).

${ }^{38}$ S. Chapman, B. C. Garrett, and W. H. Miller, J. Chem. Phys. 63, 2710 (1975). 
${ }^{39}$ C. G. Callan and S. Coleman, Phys. Rev. D 16, 1762 (1977).

${ }^{40}$ S. Andersson, G. Nyman, A. Arnaldsson, U. Manthe, and H. Jónsson, J. Phys. Chem. A 113, 4468 (2009).

${ }^{41}$ J. Cao, C. Minichino, and G. A. Voth, J. Chem. Phys. 103, 1391 (1995).

${ }^{42}$ J. Cao and G. A. Voth, J. Chem. Phys. 106, 1769 (1997).

${ }^{43}$ C. D. Schwieters and G. A. Voth, J. Chem. Phys. 108, 1055 (1998).

${ }^{44}$ C. D. Schwieters and G. A. Voth, J. Chem. Phys. 111, 2869 (1999).

${ }^{45}$ J. O. Richardson, R. Bauer, and M. Thoss, J. Chem. Phys. 143, 134115 (2015).

${ }^{46}$ J. O. Richardson, J. Chem. Phys. 143, 134116 (2015).

${ }^{47}$ E. R. Heller and J. O. Richardson, J. Chem. Phys. 152, 034106 (2020).
${ }^{48}$ C. L. Vaillant, M. J. Thapa, J. Vaníček, and J. O. Richardson, J. Chem. Phys. 151, 144111 (2019).

${ }^{49}$ T. Yamamoto, J. Chem. Phys. 33, 281 (1960).

${ }^{50}$ W. H. Miller, S. D. Schwartz, and J. W. Tromp, J. Chem. Phys. 79, 4889 (1983).

${ }^{51}$ J. W. Tromp and W. H. Miller, Faraday Discuss. 84, 441 (1987).

${ }^{52}$ B. R. Johnson, J. Comput. Phys. 13, 445 (1973).

${ }^{53}$ J. Cao and B. J. Berne, J. Chem. Phys. 92, 7531 (1990).

${ }^{54}$ J. Cao and G. A. Voth, J. Chem. Phys. 102, 3337 (1995).

${ }^{55}$ S. Yang and J. Cao, J. Chem. Phys. 122, 094108 (2005). 